\title{
Interactive Volumetric Visual Analysis of Glycogen-derived Energy Absorption in Nanometric Brain Structures
}

\author{
M. Agus ${ }^{1,3}$ and C. Calì ${ }^{2}$ and A. Al-Awami ${ }^{4,1}$ and E. Gobbetti ${ }^{3}$ and P. Magistretti ${ }^{2}$ and M. Hadwiger ${ }^{1}$ \\ ${ }^{1}$ King Abdullah University of Science and Technology (KAUST) \& Visual Computing Center (VCC), Thuwal, Saudi Arabia \\ ${ }^{2}$ King Abdullah University of Science and Technology (KAUST) \& Biological and Environmental Science and Engineering (BESE), Saudi Arabia \\ ${ }^{3}$ Center for Advanced Studies, Research, and Development in Sardinia (CRS4) / Visual Computing Group, Cagliari, Italy \\ ${ }^{4}$ Saudi Aramco \& EXPEC Computer Center, Dharhan, Saudi Arabia
}
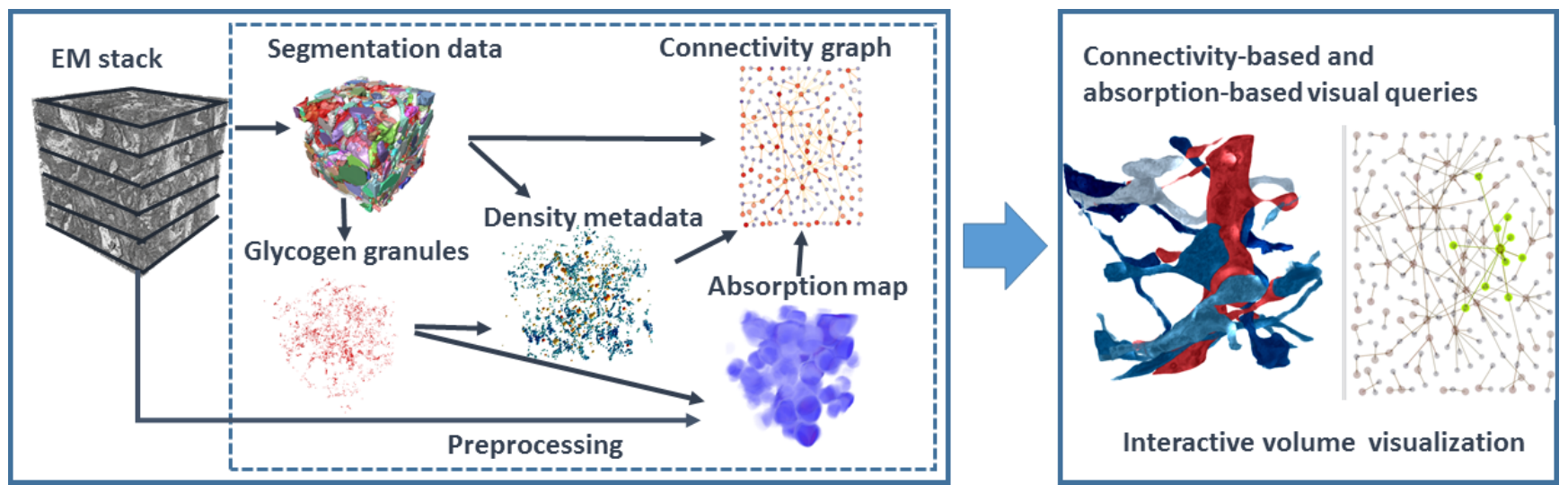

Figure 1: Pipeline overview: the proposed framework takes as input Electron Microscopy stacks representing brain cells at nanometric resolution, labelled structures, and glycogen energy sources. After computing volumetric absorption maps and abstract connectivity information, it provides real-time interactive visual exploration of brain structures augmented with absorption metadata

\begin{abstract}
Digital acquisition and processing techniques are changing the way neuroscience investigation is carried out. Emerging applications range from statistical analysis on image stacks to complex connectomics visual analysis tools targeted to develop and test hypotheses of brain development and activity. In this work, we focus on neuroenergetics, a field where neuroscientists analyze nanoscale brain morphology and relate energy consumption to glucose storage in form of glycogen granules. In order to facilitate the understanding of neuroenergetic mechanisms, we propose a novel customized pipeline for the visual analysis of nanometric-level reconstructions based on electron microscopy image data. Our framework supports analysis tasks by combining i) a scalable volume visualization architecture able to selectively render image stacks and corresponding labelled data, ii) a method for highlighting distance-based energy absorption probabilities in form of glow maps, and iii) a hybrid connectivitybased and absorption-based interactive layout representation able to support queries for selective analysis of areas of interest and potential activity within the segmented datasets. This working pipeline is currently used in a variety of studies in the neuroenergetics domain. Here, we discuss a test case in which the framework was successfully used by domain scientists for the analysis of aging effects on glycogen metabolism, extracting knowledge from a series of nanoscale brain stacks of rodents somatosensory cortex.
\end{abstract}

CCS Concepts

- Human-centered computing $\rightarrow$ Scientific visualization; $\bullet$ Applied computing $\rightarrow$ Life and medical sciences; Imaging; 


\section{Introduction}

Understanding the intimate relationship between brain morphology and its function, and the effects of their interaction in terms of behavior is one of the major challenges in medicine and biology to date. Recent progresses in digital acquisitions and analysis of biological samples are offering unprecedented possibilities of insights to neuroscientists.

Among the different digital acquisition modalities, automated serial section electron microscopy (3DEM) is raising attention in the field of neuroscience because of its level of detail in terms of resolution, as well as the high data production rate (1TB/hour) [OZ17]. Electron micrographs reach a resolution of a nanometer per pixel, revealing features ranging from full structural cellular details such as axons, dendrites and synapses (the neuropil), to smaller intracellular organelles like synaptic vesicles. For these reasons, electron micrographs are, to date, the best source for digital reconstruction of the connectivity between neurons [LPS14].

The brain and its components are complex three-dimensional structures. Gaining visual understanding of the relationships between morphological and functional aspects of brain cells is therefore of primary importance. Research in visualization for neuroscience thus strives to develop novel visual methods for enabling neuroscientists to process, explore and filter data at multiple scales, from cellular to anatomical level. In this context, many efforts have been devoted to develop minable brain maps and to reconstruct the brain wiring and connections among individual neuron to infer networks inside and among functional areas [HCT*17,ZLP*18].

Recent advances in brain imaging allow scientists to extend this approach to brain metabolism. In particular, current processing pipelines make it possible to detect energy sources at nanoscale level [CBB*16]. Specifically, glycogen granules are energy-storage molecules, whose morphology is similar to spheres with a diameter roughly ranging from 10 to 80 nanometers, and can be easily resolved using electron microscopy. Glycogen granules are stored in astrocytes, a type of brain cell whose thousands of cellular processes are in contact with tenth of thousands of synapses [Cal17] to sustain their metabolism, which is critical during learning and memorization [MA15]. Understanding and quantifying their distribution is of particular relevance for neuroscientists, in order to grasp where and when neurons use energy under this form [OBA*16, CTM19]. Hence, sophisticated tools for visual analysis of neural structures and energy sources are required for improving ultrastructural analysis and driving speculations about energy consumption during neural activity. To this end, some frameworks have been very recently proposed to support neuroscientists. They are targeted to provide specific abstract representations built around glial cellular structures [MAAB*18], or to improve visual perception of the influence of energy sources on cellular surface reconstructions through immersive environments [ABG* 18]. Current approaches, however, do not target general analysis tasks in the neuroenergetics domain.

We propose here a novel visual analysis framework, built around 3DEM image data, label volumes and connectivity metadata, targeted to real time interactive exploration of volume stacks. The framework integrates the following components:
- an illustrative absorption map, modelling the energy absorption in volumes, and computed on EM data according to the spatial distribution of energy sources;

- a graph-based layout representation of neurites and energy sources, which can be used for selective absorption-based visual queries;

- a scalable interactive volume visualization system, integrating the absorption maps, the segmented volume and the graph layout, providing effective ways to perform real time inspection of the potential effects of glycogen energy sources over specific neural structures.

To the best of our knowledge, it is the first system using volumetric data for absorption-based metabolic analysis, as well as to employ density-based visual queries for selective visualizations of electron micrographs representing nanometric brain structures. In addition to creating visually compelling real-time explorations at various scales of highly detailed cellular structures, the proposed framework provides neuroscientists tools for selective absorptionbased and connectivity-based queries that can be used for testing energy metabolism hypothesis, performing correlative analysis and datasets comparisons, and driving and filtering time-consuming statistical analysis.

The framework is currently successfully used for analysis on micrographs acquired from rodent brain samples [CWB*18b]. We report here on the glycogen analysis of aging effects on mice brains, performed on six animals (three young and three old), and we show how the visual framework help domain scientists in highlighting the differences between the two groups, and guide them in performing comparisons, and testing the metabolic hypothesis.

\section{Related work}

The proposed framework introduces, combines, and extends results in interactive and illustrative volume rendering, as well as in visual analysis of nanoscale neural structures. A complete overview of the related work is out of scope. In the following, we discuss the literature most directly related to our method, referring the reader to established surveys for more general information.

Volume rendering of Electron Microscopy data. Direct volume ray casting is a popular technique used for real-time interactive visualization of $3 \mathrm{D}$ image stacks. Its most common implementation approximates the rendering equation in order to simulate absorption and emission effects and represent optical properties for revealing external and internal structures. It has been applied successfully for exploration of data coming from different application domains. We refer the reader to established reviews on GPU based large scale volume visualization [BHP15] and on compression-domain approaches [BRGIG* 14 ] for a general overview of the field. With respect to direct rendering of volume electron microscopy data, Hadwiger et al. [HBJP12] presented the first system that enabled neuroscientists to interactively explore petascale volume data resulting from high-throughput electron microscopy data streams. Brix et al. [BPH14] introduced a method targeted to visualization of multichannel microscopy data streams. Recently Sarton et al. [SCRL16] presented an approach for high capacity screening of slices based 
on a tool combining pyramidal images representation and $3 \mathrm{D}$ visualization on multi-stereo display, in order to simulate virtual microscopy (VM). Finally, Borboor et al. [BJA*19] proposed a workflow for the visualization of neuronal structures in wide-field microscopy images of brain samples, which uses a gradient-based distance transform and multi-scale enhancement filters. Our system comprises a scalable volume rendering component extending the method presented by Hadwiger et al. [HBJP12], and integrating an hybrid object-based empty space skipping scheme [HAAB $\left.{ }^{*} 18\right]$ and a data structure for hybrid culling of integer label data [BMA*19], in order to enable real-time inspection of volumetric EM data together with label data and additional metadata.

Illustrative methods on heterogeneous medical volume data. Various methods have been presented during the last decade for providing high-quality illustrative and non-photorealistic rendering of volume data in order to highlight structures and features of interest. We refer the reader to classic surveys for a coverage of illumination methods applied to volume rendering [JSYR12, DRN*17] and of perceptually motivated methods for visualization of medical volume data $\left[\mathrm{PBC}^{*} 16\right]$. With respect to methods for highlighting structures and functional activity in volume data, Nguyen et al. [NOE* 10$]$ proposed a method for concurrent volume visualization of functional Magnetic Resonance Imaging (fMRI), in which the fMRI signal is treated as light emission when rendering a patient-specific high resolution reference MRI volume, and rendered as light glowing from active regions. Lawonn et al. [LSPV15] introduced an illustrative 3D visualization technique, specifically designed for use with PET/CT datasets, and targeted to the detection of foci in the PET data and to localize these regions by providing anatomical contextual information from the CT data. Similarly, Merten et al. [MGLS*16], proposed an illustrative rendering pipeline which combines anatomical information from CT scans with functional information from PET scans, by using order independent transparencies together with boundary enhancements and silhouettes. Our method treats brain glycogen granules as energy sources and computes glow maps by considering an absorption-based Monte Carlo integration scheme over the electron microscopy volume data. The absorption map provides local proximity information of energy sources over neurites, and can be used for visual correlative analysis, in order to understand which areas are more likely to use glycogen-derived energy.

Visual analysis of neuroscience data. In the last decade, given the dramatic performance improvement of data processing and rendering techniques, a number of frameworks and methods have been proposed for helping neuroscientists analyzing brain data at various spatial and temporal scales. Bruckner et al. [BSG* ${ }^{*}$ 9] proposed a system for visual inspection of volume data of fruit fly brains acquired through confocal microscopy. Given the availability of whole brain connectivity data of fruit flies, recently various efforts have been carried out to create interactive systems for connectivity analysis [SBS* $\left.13, \mathrm{SMB}^{*} 17\right]$. By contrast, we focus here on serial section electron microscopy images at nanometric resolution, since they are able to reveal intracellular details that are fundamental to understand neuro-energetic processes [LPS14]. In general, the main challenge is to develop tools for extracting visual information from large-scale image data. To this end, Beyer

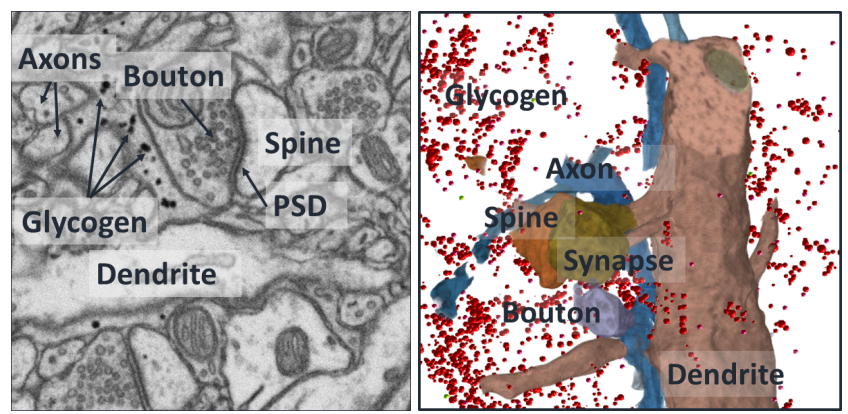

Figure 2: Neurites from nanoscale reconstruction. Left: domain scientists interpret and recognize neural structures from electron micrographs. Right: manual or semiautomatic processing provides $3 D$ reconstruction of neurites.

et al. [BAAK $\left.{ }^{*} 13\right]$ presented a volume visualization framework for extracting and filtering brain structures according to a query algebra able to create set of objects with respect to connectivity and regions of interest. The framework was moreover extended by providing visual tools for representing connectivity information by using subway lines metaphors [AABS*14], and visual tools in forms of blocks for tracking the segmentation and proof-reading for large projects [AABH*16]. Similarly, techniques for exploring 2D abstract representations of neural structures have been recently introduced to help neuroscientists in correlation analysis of morphology [MAAB $\left.{ }^{*} 18\right]$, while methods for analyzing absorption patterns have been proposed to enrich dense and sparse surface reconstructions of rodent brain structures $\left[\mathrm{ABG}^{*} 18\right]$. Our solution extends a volume visualization framework [HBJP12] with a method for highlighting local absorption patterns and tools for performing connection based and absorption based queries, able to provide neuroscientists visual hints for comparative and correlative analysis of morphology structures and glycogen distributions.

\section{Overview}

The design of our method has taken into account requirements gathered from domain experts. Scientists with high experience and reputation in the neuroenergetics field [MA15] were actively involved in all phases of the project, starting from the development of the main ideas, to the requirement analysis, to system design, and evaluation. Sec. 3.1 briefly illustrates the application domain, the design process, and the derived requirements, while Sec. 3.2 provides a general overview of our approach, justifying the design decisions in relation to the requirements. An evaluation of the method, carried out in the context of the glycogen analysis of aging effects on mice brains is presented in Sec. 6

\subsection{Requirements for nanoscale neurometabolism analysis}

We carried out a detailed task analysis in order to identify the essential analysis components. The analysis involved a review of existing documentation and literature, previous software analysis, interviews with experienced operators, as well as direct observation of 
analysis procedures being performed by domain experts. In the following, we briefly summarize the domain knowledge and the specific procedures currently used for performing ultrastructural analysis targeted to understanding neuroenergetic phenomena.

Data sources and labeling. Domain scientists perform visual and statistical analysis by carrying out dense and sparse reconstructions from electron microscopy images (Fig 2 left). According to the way digital acquisition is performed, glucose energy storage in form of glycogen granules are semi or fully automatically recognizable from micrographs.

Structures. Once neural cells and sub-parts are labelled, neuroscientists perform ultrastructural analysis by studying the morphology of the following items (Fig. 2 right):

- Neural structures: namely axons and dendrites, which are the terminals respectively sending and receiving the electric signal through boutons and spines. Boutons and spines are linked and form synapses.

- Glia structures: neuroscientists mainly focus on astrocytes, which are metabolically involved in feeding neurons and contain glycogen granules.

- Organelles: domain scientists mainly focus on mitochondria, which are contained in axons, dendrites, and astrocytes and contain the machinery for chemical transformations, included the conversion of glucose in lactate, which is the form of energy needed by neural processes.

General analysis tasks. Neuroscientists are interested in studying all possible relationships between the aforementioned structures, and perform analysis on glycogen distribution and correlation analysis with respect to synapses as well as other neural structures. The investigation efforts are mainly focused in understanding the metabolic mechanisms of memory formation, and relative degeneration, which are implemented with the creation of new synapses, maintenance, and destruction. Hence, various questions are faced, related to the spatial distribution of glycogen granules with respect to synapses, spines, boutons, dendrites and axons.

Derived requirements. After a long-term interaction process with domain scientists, the following general requirements emerged for performing visual analysis on electron microscopy images representing nanometric scale rodent brain morphology:

- R1. Correlative analysis: domain scientists are interested in finding visual and statistical correlations between various kind of neural structures;

- R2. Morphology exploration: domain scientists need to discriminate and explore morphology at various scales and details, ranging from macro-scale to figure out extents and mutual spatial relationships, to meso-scale and micro-scale, to highlight and accurately analyze finest details;

- R3. Metabolic consumption hypothesis formulation and testing: domain scientists need visual tools for formulating and testing hypothesis on metabolic consumption;

- R4. Connectivity analysis: domain scientists need to know connectivity information between various neurites. With respect to synapses, connectivity information relating spines (part of dendrites) and boutons (part of axons), are needed to understand metabolic paths;

- R5. Absorption analysis: domain scientists need to measure and classify neural objects with respect to local and global absorption patterns;

- R6. Spatial discrimination: domain scientists need to understand 3D layouts and to perform depth ordering between the different neural structures.

\subsection{Proposed framework design}

On the basis of the requirement analysis, contrary to other systems $\left[\mathrm{ABG}^{*} 18, \mathrm{MAAB}^{*} 18\right]$, which are designed around surface representations coming out of careful segmentation processes, we decided to employ and enrich volumetric representations. This is because, in general, domain scientists feel more comfortable with raw EM data, in which they can exploit their expertise for recognizing small-scale specific features that cannot be easily highlighted in surface reconstructions (for example bouton vesicles and mitochondria). Moreover, with volumetric representations, experts can perform proofreading to check that structures are correctly labelled, and to ensure that features accurately map with the original EM data. Finally, volumetric representations provide us a convenient discretization to accurately compute and visually represent absorption information to be mapped not only over the membranes $\left[\mathrm{ABG}^{*} 18\right]$, but also in the interior part of the neural structures to be analyzed. As a result of this decision, we considered the previous requirements for designing and implementing the visualization framework, and we chose and integrated the following main components:

- Scalable hybrid volume rendering framework: by composing raw image data together with labelling information about neural objects, we developed a scalable volume visualization architecture able to fulfill requirements R2, R5, and R6;

- Radiance-based metabolic consumption glow map: by mapping the energy absorption on volume data, our system delivers to neuroscientists additional visual information able to fulfill requirements R1, R3, and R6;

- Graph-based connectivity and absorption information: by integrating metadata representing connectivity with neural structures and spatial geometric information relating energy sources and neural structures, our framework can provide to domain scientists the capability to perform extended absorption and connectivity queries able to fulfill requirements R1, R4, and R5.

As illustrated in Fig. 1, the framework can be decomposed into an offline processing pipeline, which transforms raw EM data into higher-level structures, and a run-time visualization system, which exploits the precomputed structures to provide real-time interaction with data. In the following sections, we will first detail the offline processing pipeline (Sec. 4), and then discuss how the precomputed data is integrated in our novel visual analysis framework (Sec. 5).

\section{Offline processing pipeline}

The input for the framework is a stack of 8-bit images acquired with electron microscope at nanoscale resolution and representing micro-scale portions of the brains. The processing pipeline is 
composed by a sequence of steps that are carried out to extract geometry, connectivity, density and absorption information related to neural processes (specifically axons, spines, dendrites, boutons and synapses), cells (astrocytes and neurons), and compounds of interests (glycogen granules) contained inside the brain samples. The full pipeline is schematized in Fig. 1 left.

Segmentation data. Starting from electron microscopy images, generating labels which associate each pixel to a specific neural structure is a general task performed by domain scientists to support ultra-structural studies. In this work, we considered a hybrid pipeline [CCK*18] involving a first, rough automatic segmentation that runs offline, performed through iLastik tool [SSKH11], for finding the gross features and processes of a cell. This first segmentation is then followed by a manual proofreading phase, performed through TrackEm2 tool [CSS*12], for specifying exact boundaries and finer details. Given a stack of images representing a brain sample, the output of this processing task is a dense reconstruction containing the following objects: glycogen granules (which are represented by spheres of radii varying between 10 and $50 \mathrm{~nm}$ ), astrocyte processes, neural processes (dendrites, axons, spines and boutons) and synapses.
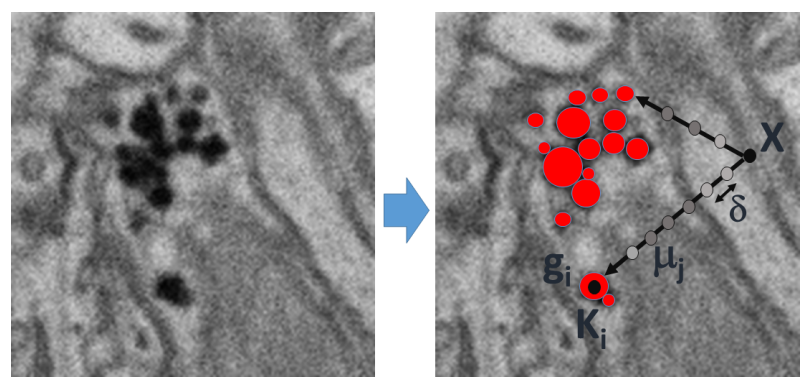

Figure 3: Absorption map computation: for each position $x$, we compute energy contributions from neighbor glycogen granules. We use a Russian roulette scheme for sampling density values along directions connecting $x$ to the glycogen granules.

Absorption maps. We compute local absorption maps by considering as input the EM image stacks and the collection of glycogen granules obtained through segmentation, and by using a geometrybased absorption model consisting of a Gaussian-based extinction decay and a density-based function. The objective of the model is to derive a scalar function representing for each position of the volume the potential absorbed energy derived from glycogen granules. To this end, we extend to volumes the formulation proposed by Agus et al. [ABG*18], originally derived for computing absorption on surface reconstructions. By considering a simplified version of the radiance transfer equation, we model the effect of glycogen on surrounding structures with an absorption-only mechanism [ZL13]. Specifically, we define for each position the absorption value $\lambda(x)$ as the sum of the energy contribution from glycogen granules, obtained by Monte Carlo integration on EM density values (ranging from 0 to 255 in 8-bit images). Given the list of glycogen granules $G=\left\{\mathbf{g}_{\mathbf{i}}, r_{i}\right\}$, for each position $x$ in the volume, we compute the absorption value $\lambda(x)$ related to the distribution of glycogen granules and the density of the volume, with the following equation:

$$
\lambda(x)=\sum_{i} K_{i} e^{-\int_{g_{i}}^{x} \mu(\tau) d \tau},
$$

where $K_{i}=K \frac{4}{3} \pi r_{i}^{3}$ is the glucose content associated to each glycogen granule (up to $30 \mathrm{~K}$ glucose units and proportional to the volume of the granule [MA15]), and $\mu(\tau)$ is the extinction function, which depends on density values $d(\tau)$ contained in electron microscope images. In our case, we made the assumption that membranes, which are represented by low EM density values mostly absorb energy. So, we considered an extinction function which is inversely proportional to the EM density value $\left(\mu(\tau)=1-\frac{d(\tau)}{255}\right)$. In order to rapidly approximate equation 1 , for each position $x$ in the volume, we use a Kd-tree for recovering the glycogen granules which can provide energy to that position. We then approximate the line integral by sampling the direction connecting the glycogen granule with a Russian roulette scheme that generates a different number of samples $\mathrm{N}$ for each direction:

$$
\int_{g_{i}}^{x} \mu(\tau) d \tau \approx \delta \sum_{j=1}^{N} \mu_{j}=\delta \sum_{j=1}^{N} \mu\left(g_{i}+j \delta \cdot \frac{x-g_{i}}{\left\|x-g_{i}\right\|}\right),
$$

where $\delta=\frac{\left\|x-g_{i}\right\|}{N}$ is the distance between consecutive samples along the line (see figure 3 for a simplified scheme of the absorption map computation). In this way we are able to take into account different energy propagation directions and the density variability inside the brain structures. The final output of this process is a stack

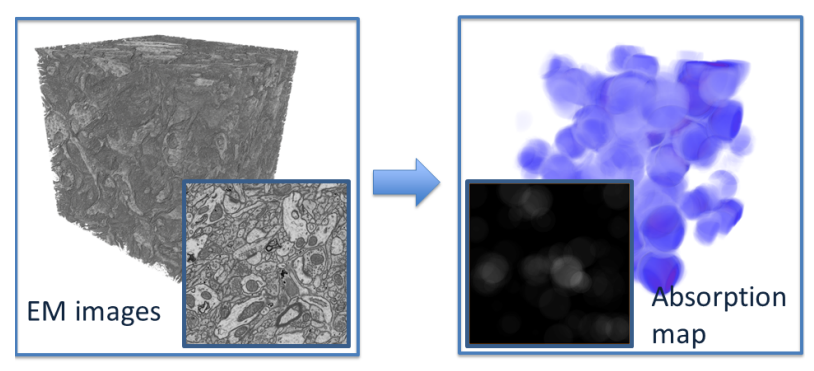

Figure 4: Absorption map: the output of computation is a volume of absorption values at the same resolution of the input EM stack.

of images at the same resolution of the original EM stacks, containing the computed absorption values (see figure 4). For all results in the paper we used $K=10.0$ for glycogen energy and $N=10$ for the Russian roulette scheme.

Density metadata. Density metadata contains information about spatial distribution of objects. In the context of a glycogen analysis framework, domain scientists are interested in density information related to clusters formed by glycogen granules, and how to they relate to other objects with compact size that can be represented as spatial distributions, namely synapses. To obtain this information, we use a Kd-tree containing all glycogen granules, and we compute densities associated to either glycogen granules or synapses. In this way, we get $3 \mathrm{D}$ spatial distributions of glycogen granules and synapses that provide useful reference during interactive analysis of volumetric data. We color-map glycogen granules with respect to 


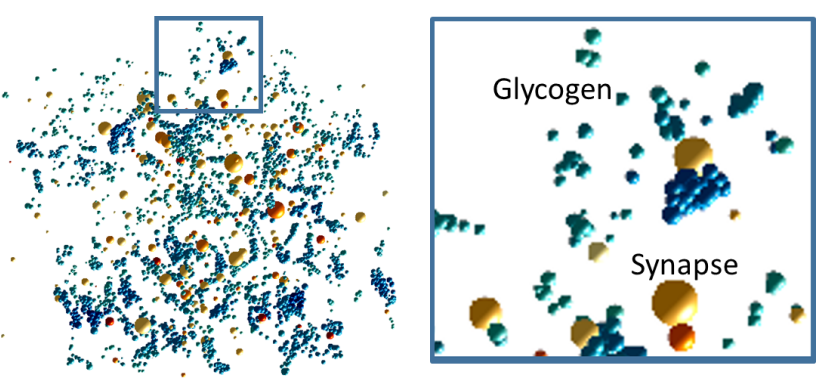

Figure 5: Glycogen and synapse distribution: spatial information about glycogen and synapse distribution can be useful to understand absorption pattern in the various neurites. We color map glycogen granules and synapses according to glycogen density values.

density values, while we represent synapses as spheres with radius proportional to the dimension and color depending on the density values. Figure 5 shows an example of glycogen and synapse distribution that is used during interactive analysis.
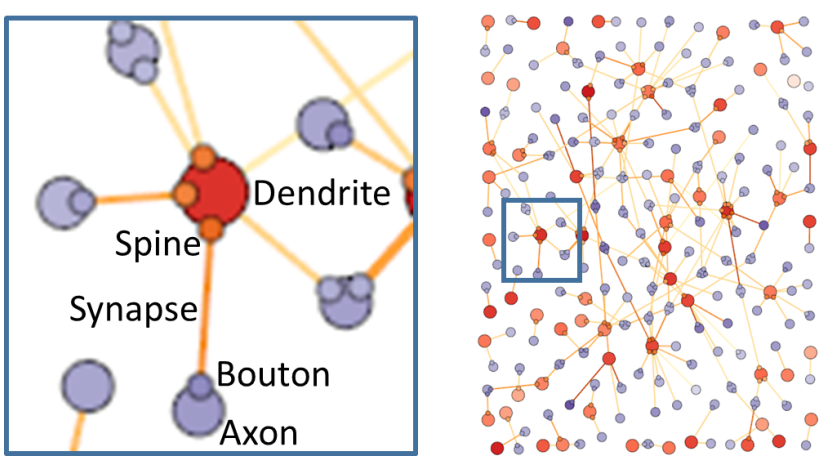

Figure 6: Connection representation: we use a node-link $2 D$ graph to represent the connections between the different neural structures. Edges represent synapses, while main nodes represent dendrites and axons. We also include small nodes to represent spine and boutons. We color-map all elements according to the energy absorption values $\lambda_{i}$ as computed through the model in equation 1.

Connectivity metadata. Connectivity metadata contain information about how the neurites are connected. Specifically to which bouton of which axon and to which spine of which dendrite every synapse is connected. To obtain this metadata, we carry out a geometry analysis based on dense reconstruction. The method takes as input the segmented neurites, and the synapse spatial distribution. For each synapse, which represents a connection between neural objects, we estimate the closest neural objects by representing them through oriented bounding boxes and by inserting them in Kd-trees for speeding up overlap computations. Moreover, we associate absorption metadata to neurites by integrating information provided by labelled images and absorption maps. Specifically, for each cellular structure represented by a specific label (namely axons, dendrites and their respective boutons and spines), we derive a total absorption value by summing up all the voxel absorption contributions computed according equation 1 . The output of this process are absorption values $\lambda_{i}$ for all objects, which provide clear indication about the importance of the various neural structures, in a way to highlight potential regions of interest during analysis. Thus, we use connectivity information together with absorption metadata associated to neural objects to derive a node-link 2D representation which provides an effective and minable map for exploring and analyzing the dataset. In order to have a clear and significant graph representation we considered an energy-based layout scheme using a stochastic method [DH96, ZPG17, ZPG17, HWP*18]. Given a node-link representation $\mathcal{G}=\{\mathcal{V}, \mathcal{E}\}$ where $\mathcal{V}=\left\{v_{i}: v_{i}=\left(x_{i}, y_{i}\right), i=1 . . N\right\}$ are the nodes represented by $2 \mathrm{D}$ positions, and $\mathcal{E}=\left\{\varepsilon_{i j}:\left(v_{i}, v_{j}\right) \in\right.$ $\mathcal{V}\}$ are the edges connecting the nodes, our algorithm tries to find the optimal layout $\mathcal{V}_{\text {opt }}$ by using an energy-based model. Specifically, we consider a cost function composed by the following contributions:

- a penalization component inversely proportional to mutual distances which repulses all nodes in a way to avoid overlapping. For each node, we find the K-nearest-neighbor nodes, and we accumulate the weighted distances:

$$
E_{R}(\mathcal{V})=\sum_{v_{i} \in \mathcal{V}} \sum_{v_{j} \in K N N\left(v_{i}\right)} \frac{w_{i j}}{d_{i j}}
$$

where weights depend on absorption values of the objects involved (in our case $w_{i j}=\lambda_{i} \lambda_{j}$ );

- a penalization component directly proportional to the squared distance of connected nodes, which tries to reduce the length of graph edges:

$$
E_{A}(\mathcal{E})=\sum_{\varepsilon_{(i, j)} \in \mathcal{E}} w_{i j} d_{i j}^{2}
$$

where weights depend on absorption values of the objects involved (in our case $w_{i j}=\lambda_{i} \lambda_{j}$ );

- a penalization component which tries to reduce the crossing edges in the layout:

$$
E_{C}(\mathcal{E})=\sum_{\varepsilon_{i j} \in \mathcal{E}} \sum_{\varepsilon_{l m} \in \mathcal{E}} w_{i j} w_{l m} \chi\left(\varepsilon_{i j}, \varepsilon_{l m}\right),
$$

where

$$
\chi\left(\varepsilon_{i j}, \varepsilon_{k l}\right)= \begin{cases}1, & \exists \text { intersection }\left(\varepsilon_{i j}, \varepsilon_{k l}\right) \\ 0, & \text { otherwise }\end{cases}
$$

and weights depend on absorption values of the objects involved (in our case $w_{i j}=\lambda_{i} \lambda_{j}$ and $w_{k l}=\lambda_{k} \lambda_{l}$ ).

Hence, the total cost associated to a node-link representation $\mathcal{G}=$ $\{\mathcal{V}, \mathcal{E}\}$ is

$$
E(\mathcal{G})=\rho_{A} E_{A}(\mathcal{E})+\rho_{R} E_{R}(\mathcal{E})+\rho_{C} E_{C}(\mathcal{E}),
$$

where $\rho_{A}, \rho_{P}, \rho_{C}$ are arbitrary weights that can be chosen to privilege given contributions, in order to have for example a more uniform node distribution or a more compact layout. In all results of this paper we considered $\rho_{A}=1000.0, \rho_{R}=0.01, \rho_{C}=5000$. To find the position of the nodes minimizing the cost layout we need to solve an optimization problem with $2 N$ variables:

$$
\mathcal{V}_{\text {opt }}=\arg \min _{\mathcal{V}} E(\mathcal{G})
$$


To this end, we use an iterative simulated annealing scheme [DH96]. To reduce the complexity, we solve a different optimization problem for each node at time, by placing nodes iteratively in the graph in batches, similarly to [ZPG17]. In this way, the graph grows while trying to keep a minimum energy status. For each insertion of batch nodes, the optimization order of the nodes is randomized, in order to try to avoid trapping in a local minimum configuration. In all results of this paper, we used batches of 20 nodes, and 5 random shuffle loops per batch insertion, and 1000 iterations per node optimization. The final layout contains principal nodes representing dendrites, and axons, color-mapped according to absorption values $\lambda_{i}$, and edges representing synapses, color-mapped according to density values. For each synapse, we also place small nodes representing boutons and spines close to the associated axon, and dendrite respectively. In figure 6 we show an example of node-link layout together with a detail inset.

\section{Interactive visual components}

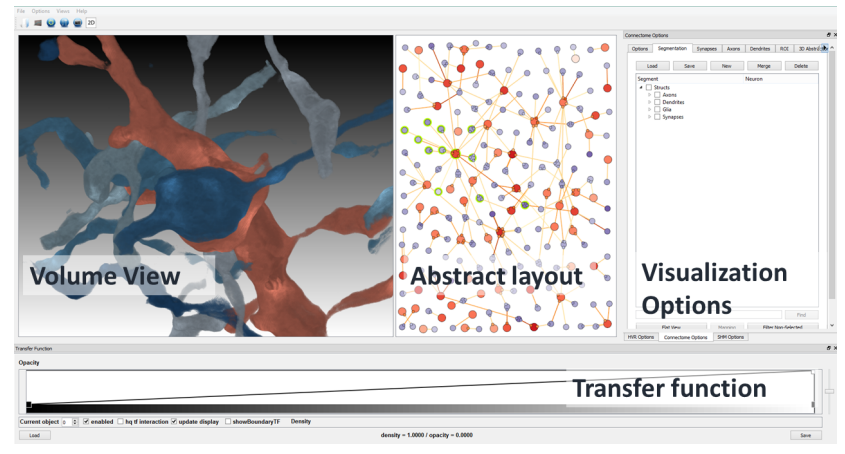

Figure 7: Visualization framework: the interactive visualization framework integrates two main views, one for real time visualization of composed volumes, and one for showing abstract layouts of neural structure connections. The GUI contains also widgets for various visualization options, and for changing transfer functions.

We used the original EM image data together with the data and metadata computed using methods in section 4 to create an interactive visualization framework for scalable real-time analysis of 3D volumetric data (see figure 7). The framework is composed of three main views:

- Volume interaction view: where we compose and render image stacks, together with metadata volume information related to spatial distributions;

- Graph interaction widget: where we show connectivity and absorption information as a 2D graph layout, with objects directly connected to the volume view for interactive selection of rendered objects;

- Visualization options widgets: where together with enabling/disabling toggles we provide standard tools for volume clipping, widgets for selection of objects as hierarchical lists, and widgets for transfer function modification.

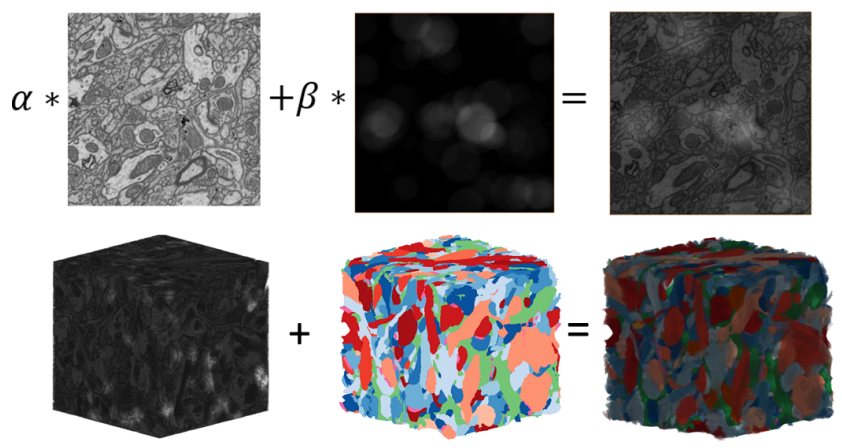

Figure 8: Volume view: absorption glow volumes are blended with original EM stacks (on the top), and the composed signal is modulated by labelled volumes for providing $3 D$ raycasted representation of objects with absorption signals (bottom view).

Volume interaction view. In the volume interaction view we incorporated and extended a real-time volume visualization scalable component able to provide interactive ray casting of image stacks up to terabyte-size [BAAK $\left.{ }^{*} 13\right]$. We integrated in a client-server rendering architecture a series of high performance strategies for dealing with massive volume data:

- a visualization-driven scalable multi-resolution memory architecture built around 2D microscope image tiles, which decouples sample access time during ray-casting from the size of the multi-resolution hierarchy [HBJP12];

- a high-performance hybrid ray casting method that scales to multiple volumes of several teravoxels each, enabling the concurrent visualization and querying of the original EM volume, additional segmentation volumes, and additional meta data comprising a variety of neuronal data attributes [BAAK $\left.{ }^{*} 13\right]$;

- a method for performing efficient empty space skipping during real time ray casting, by using an hybrid strategy that balances the computational load between determining empty ray segments in a rasterization (object-order) stage, and sampling non-empty volume data in the ray-casting (image-order) stage [HAAB $\left.{ }^{*} 18\right]$;

- a method for performing efficient culling of objects and spatial queries which combines deterministic and probabilistic representations of label data in a data-adaptive hierarchical data structure [BMA*19].
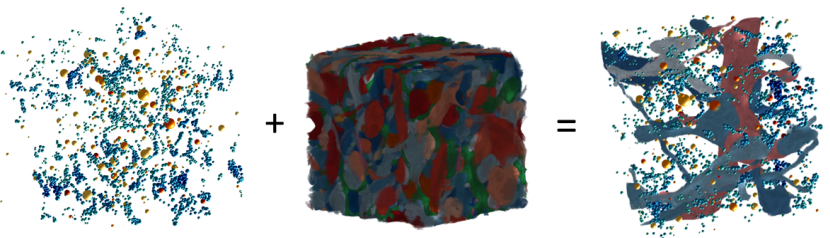

Figure 9: Spatial information: glycogen and synapses distribution provide references for highlighting the areas of interests during selective analysis.

With the volume interaction view, users can explore in real-time a 
semitransparent representation of original EM data together with the absorption glow maps and with the label data. Specifically, we composed the absorption glow map with the EM original data by defining two weights $\alpha$ and $\beta$ for creating a blended image which keeps the original membrane structure and over imposes the absorption signal (see figure 8 top). In all results of the paper we used $\alpha=0.25$ and $\beta=0.75$. Since the segmentation volume provides different colors for each brain cellular structure, we blended it with the composed absorption plus EM image signal in a way to enable users to interactively select the objects to render and visualize all signals concurrently (see figure 8 bottom). Finally, we provided spatial distribution of glycogen granules and synapses as visual references for helping domain scientists individuating areas of interest and selecting objects to visualize and explore (see figure 9).
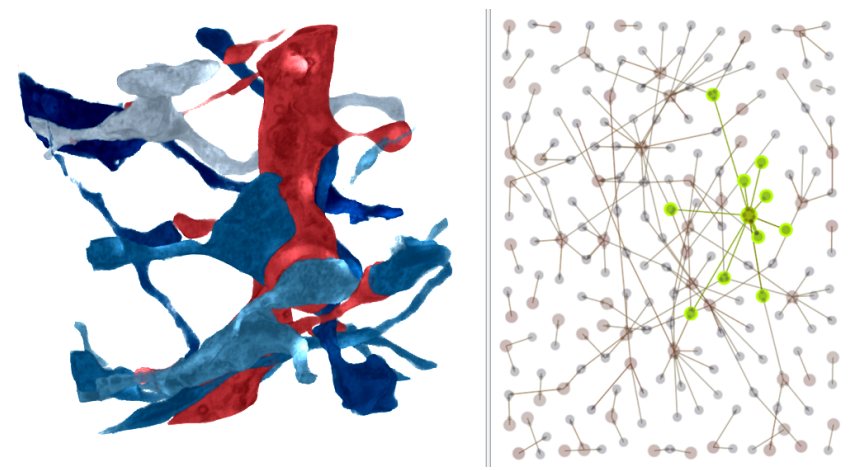

Figure 10: Graph interaction: different rendering patterns can be created by selecting objects in the graph widget. Connected objects can be selected and visualized together.

Graph interaction widget. The 2D node-link abstract layout computed in preprocessing associates each node to a specific labelled neural object in the volume view, and can be used to interactively select the objects to render. We color mapped nodes and edges according to absorption values, so domain scientists can select at a glance the objects with higher absorption and focus on the areas where it is supposed to find the most interesting metabolic phenomena. In all results provided in this paper we used color maps from ColorBrewer [HB03]: single hue Blues for axons and boutons, single hue Reds for dendrites and spines, YlOrRd for synapses, and $\mathrm{GnBu}$ for glycogen granules. The graph widget allows users also to follow the connections between neural objects, and interactively create different rendering patterns, by selecting objects connected together by synapses (see Fig. 10).

\section{Results}

We implemented all preprocessing and rendering components in $\mathrm{C}++$. We separated the preprocessing components in different scripts for computing absorption volumes, density and connectivity metadata, and used on a laptop equipped with a CPU Intel i7-4700HQ running Linux Ubuntu Kernel version 3.13, and on a PC equipped with two CPU Intel Xeon $2,3 \mathrm{GHz}$ and $128 \mathrm{~GB}$ RAM and running Windows 8 . We tested the client-server rendering framework have been tested on various setups: a PC desktop equipped with two CPU Intel Xeon $2,3 \mathrm{GHz}$ and 128 GB RAM, an NVIDIA Geforce GTX Titan Black, and running Windows 8, a laptop Asus ROG G703GI equipped with an Intel Coffee Lake i98950HK 2,9Ghz, an NVIDIA GTX 1080 VR-Ready, and 512 GB RAM, running Windows 10 . We also used the laptop setup to drive a large scale tiled display wall composed by an array of 10x4 tiles for a total dimension of 33.5 feet $x 7.5$ feet and a total resolution of around 40 Mpixels. In all test conditions, the framework showed interactive performance, with frame rates in the range 10-60 fps, independently from the scale at which exploration was performed.

Data acquisition and processing. We evaluated the system on a series of datasets used for ultrastructural analysis in the neuroenergetics domain: six datasets (three 4 months old and three 24 months old), forming a complete use case about the effects of aging on glycogen in rodents brain, that we obtained from segmentation and dense reconstruction of neuropil and glycogen granules within volumes of Layer 1 somatosensory cortex mice brains [CWB*18a], from FIB-SEM stacks [TG16]. For comparing to standard systems currently used for glycogen analysis [CBB*16], we processed also a portion of hippocampus of a 2 months old rat [CBB*16]. Somatosensory cortex datasets are public domain and available as Dryad resource $\left[\mathrm{CWB}^{*} 18 \mathrm{~b}\right]$ : table 1 shows the main statistics, together with the pictorial representation of the volume view and the abstract layout view. We list data resolution, physical size, and number of segmented objects. We include also the classification of neurites for each datasets: number of spines, dendrites, synapses, boutons and axons. We add glycogen-related density statistics: number of glycogen granules, and densities of glycogen content associated to synapses. We report also on processing times for the metadata used for interactive exploration: absorption maps, density and connectivity metadata.

In the following, we provide a qualitative evaluation of the system performed by domain scientists, and a comparison from user perspective with respect to standard tools employed for ultrastructural analysis $\left[\mathrm{ABG}^{*} 18, \mathrm{CBB}^{*} 16\right]$. Finally, we discuss a use case concerning brain aging through the investigation of glycogen on aging rodents.

\subsection{Domain expert evaluation}

Since we designed and implemented the system for addressing specific exigencies related to a particular application domain, to assess the performances we relied on a qualitative evaluation performed by domain scientists. Specifically we involved two neuroscientists with high level of expertise, who are coauthoring this paper, and we asked them to perform various analysis sessions on two different setups: a single user standard desktop setup, and a multiwall collaborative setup, in which one operator directly controlled the application while domain scientists could lead the session through voice commands and discuss and speculate about the features visualized (see the accompanying videos).

Procedure. During the analysis sessions, we observed the behavior and performances of domain experts and we carried out live interviews. Users followed various strategies for inspecting the data (see the accompanying videos). Nonetheless, in most cases 
Table 1: Data set statistics: for the brain samples used in Sec. 6 [CWB* 18b]. We list data resolution, physical size, and number of segmented objects. We include also the classification of neurites for each datasets: number of spines, dendrites, synapses, boutons and axons. We add glycogen-derived absorption statistics: number of glycogen granules, and average and maximum glycogen density information related to synapses. Finally we add processing times for absorption maps and metadata computation.

\begin{tabular}{|c|c|c|c|c|c|}
\hline \multirow[t]{8}{*}{ Dataset } & & Description & Classification & Metadata statistics & Processing times \\
\hline & 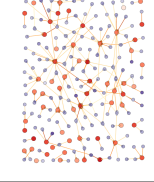 & $\begin{array}{c}\text { Mouse cortex } 1 \\
4 \text { months } \\
831 \times 831 \times 280 \\
5 \mu m \times 5 \mu m \times 5 \mu m\end{array}$ & $\begin{array}{l}\text { Synapses: } 189 \\
\text { Spines: } 79 \\
\text { Dendrites: } 66 \\
\text { Boutons: } 115 \\
\text { Axons: } 274\end{array}$ & $\begin{array}{l}\text { Glycogen granules: } 3040 \\
\text { Syn/Gly avg density }\left(\mathrm{nm}^{3}\right): 13.4 \mathrm{~K} \\
\text { Syn/Gly max density }\left(\mathrm{nm}^{3}\right): 70.4 \mathrm{~K}\end{array}$ & $\begin{array}{l}\text { Absorption: } 3 \mathrm{~m} 40 \mathrm{~s} \\
\text { Density: } 49 \mathrm{~s} \\
\text { Connectivity: } 2 \mathrm{~h} 28 \mathrm{~m}\end{array}$ \\
\hline & $\begin{array}{l}2 \\
\cdots\end{array}$ & $\begin{array}{c}\text { Mouse cortex } 2 \\
24 \text { months } \\
833 \times 833 \times 468 \\
5 \mu m \times 5 \mu m \times 5 \mu m\end{array}$ & $\begin{array}{l}\text { Synapses: } 212 \\
\text { Spines: } 104 \\
\text { Dendrites: } 84 \\
\text { Boutons: } 102 \\
\text { Axons: } 381\end{array}$ & $\begin{array}{l}\text { Glycogen granules: } 7247 \\
\text { Syn/Gly avg density }\left(\mathrm{nm}^{3}\right): 10.4 \mathrm{~K} \\
\text { Syn/Gly max density }\left(\mathrm{nm}^{3}\right): 50.8 \mathrm{~K}\end{array}$ & $\begin{array}{l}\text { Absorption: } 4 \mathrm{~m} 34 \mathrm{~s} \\
\text { Density: } 2 \mathrm{~m} 19 \mathrm{~s} \\
\text { Connectivity: } 2 \mathrm{~h} 9 \mathrm{~m}\end{array}$ \\
\hline & 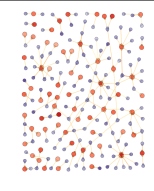 & $\begin{array}{c}\text { Mouse cortex } 3 \\
4 \text { months } \\
999 \times 999 \times 449 \\
5 \mu m \times 5 \mu m \times 5 \mu m\end{array}$ & $\begin{array}{l}\text { Synapses: } 167 \\
\text { Spines: } 152 \\
\text { Dendrites: } 81 \\
\text { Boutons: } 237 \\
\text { Axons: } 274\end{array}$ & $\begin{array}{l}\text { Glycogen granules: } 4145 \\
\text { Syn/Gly avg density }\left(\mathrm{nm}^{3}\right): 6.3 \mathrm{~K} \\
\text { Syn/Gly max density }\left(\mathrm{nm}^{3}\right): 25.5 \mathrm{~K}\end{array}$ & $\begin{array}{l}\text { Absorption: } 12 \mathrm{~m} 38 \mathrm{~s} \\
\text { Density: } 1 \mathrm{~m} 30 \mathrm{~s} \\
\text { Connectivity: } 53 \mathrm{~m} 21 \mathrm{sec}\end{array}$ \\
\hline & \begin{tabular}{l}
$a$ \\
\hdashline \\
\hdashline$\cdots \cdots$
\end{tabular} & $\begin{array}{c}\text { Mouse cortex } 4 \\
24 \text { months } \\
1001 \times 1001 \times 304 \\
5 \mu m \times 5 \mu m \times 5 \mu m\end{array}$ & $\begin{array}{l}\text { Synapses: } 185 \\
\text { Spines: } 81 \\
\text { Dendrites: } 87 \\
\text { Boutons: } 94 \\
\text { Axons: } 285\end{array}$ & $\begin{array}{l}\text { Glycogen granules: } 7055 \\
\text { Syn/Gly avg density }\left(\mathrm{nm}^{3}\right): 25.0 \mathrm{~K} \\
\text { Syn/Gly max density }\left(\mathrm{nm}^{3}\right): 121.7 \mathrm{~K}\end{array}$ & $\begin{array}{l}\text { Absorption: } 11 \mathrm{~m} 45 \mathrm{~s} \\
\text { Density: } 3 \mathrm{~m} 43 \mathrm{~s} \\
\text { Connectivity: } 1 \mathrm{~h} 25 \mathrm{~m}\end{array}$ \\
\hline & 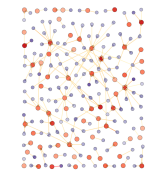 & $\begin{array}{c}\text { Mouse cortex } 5 \\
24 \text { months } \\
1001 \times 1001 \times 464 \\
5 \mu m \times 5 \mu m \times 5 \mu m\end{array}$ & $\begin{array}{l}\text { Synapses: } 171 \\
\text { Spines: } 103 \\
\text { Dendrites: } 74 \\
\text { Boutons: } 82 \\
\text { Axons: } 355\end{array}$ & $\begin{array}{l}\text { Glycogen granules: } 2436 \\
\text { Syn/Gly avg density }\left(\mathrm{nm}^{3}\right): 4.2 \mathrm{~K} \\
\text { Syn/Gly max density }\left(\mathrm{nm}^{3}\right): 23.4 \mathrm{~K}\end{array}$ & $\begin{array}{l}\text { Absorption: } 8 \mathrm{~m} 6 \mathrm{~s} \\
\text { Density: } 1 \mathrm{~m} 2 \mathrm{~s} \\
\text { Connectivity: } 1 \mathrm{~h} 10 \mathrm{~m}\end{array}$ \\
\hline & and & $\begin{array}{c}\text { Mouse cortex } 6 \\
4 \text { months } \\
998 \times 998 \times 268 \\
5 \mu m \times 5 \mu m \times 5 \mu m\end{array}$ & $\begin{array}{l}\text { Synapses: } 211 \\
\text { Spines: } 135 \\
\text { Dendrites: } 85 \\
\text { Boutons: } 102 \\
\text { Axons: } 286\end{array}$ & $\begin{array}{l}\text { Glycogen granules: } 11642 \\
\text { Syn/Gly avg density }\left(\mathrm{nm}^{3}\right): 55.1 \mathrm{~K} \\
\text { Syn/Gly max density }\left(\mathrm{nm}^{3}\right): 221 \mathrm{~K}\end{array}$ & $\begin{array}{l}\text { Absorption: } 13 \mathrm{~m} 57 \mathrm{~s} \\
\text { Density: } 3 \mathrm{~m} 20 \mathrm{~s} \\
\text { Connectivity: } 2 \mathrm{~h} 37 \mathrm{~m}\end{array}$ \\
\hline & & $\begin{array}{l}\text { Rat Hippocampus } \\
2 \text { months } \\
1178 \times 1125 \times 789 \\
7 \mu m \times 7 \mu m \times 5 \mu m\end{array}$ & $\begin{array}{l}\text { Synapses: } 93 \\
\text { Spines: } 60 \\
\text { Dendrites: } 10 \\
\text { Boutons: } 27 \\
\text { Axons: } 161\end{array}$ & $\begin{array}{l}\text { Glycogen granules: } 1847 \\
\text { Syn/Gly avg density }\left(\mathrm{nm}^{3}\right): 1.1 \mathrm{~K} \\
\text { Syn/Gly max density }\left(\mathrm{nm}^{3}\right): 7.8 \mathrm{~K}\end{array}$ & $\begin{array}{l}\text { Absorption: } 17 \mathrm{~m} 37 \mathrm{~s} \\
\text { Density: } 24 \mathrm{~s} \\
\text { Connectivity: } 6 \mathrm{~m} 29 \mathrm{~s}\end{array}$ \\
\hline
\end{tabular}

we could recognize that they performed analysis by executing the following tasks:

- Density visual assessment: neuroscientists looked for and focused on areas with high glycogen content. They used density colors of glycogen granules as visual hints to locate areas with patchy or uniform distribution [OBA*16];

- Global and local absorption analysis: for each glycogen area, neuroscientists analyzed absorption patterns related to neighboring neural structures (either dendrites or axons or synapses). They used absorption glow-maps for exploring specific features;

- Connectivity analysis: for a given neural structure of interest, users tracked the connected components (dendrites connected through spines by synapses to boutons belonging to axons), and compared different local absorption patterns associated to them.

Domain experts did non respect a specific order of operations, but their main target was to check the features of known absorption patterns or to discover new ones. Once they recognized an analyzed a pattern, they annotated it to subsequently check whether it could show up in other datasets.

Discussion. In general, domain scientists appreciated the features provided by the framework, and they specifically pointed out:

- immediate and easy to understand representation of energy source distribution and global and local absorption patterns; 

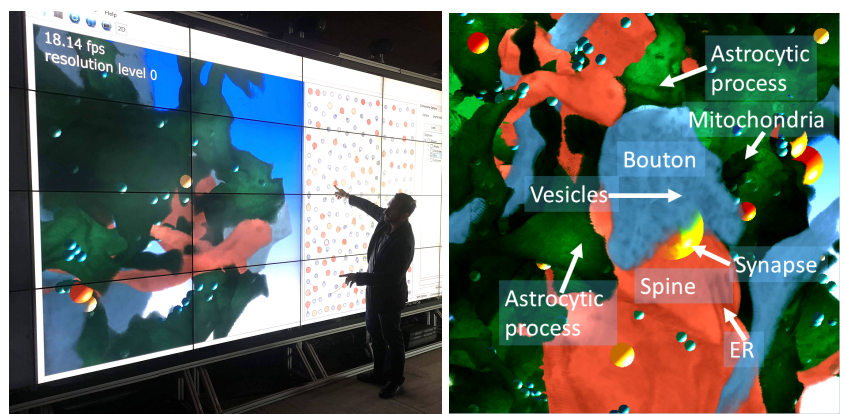

Figure 11: System evaluation. Left: expert neuroscientist performs analysis sessions by using a large scale tiled display wall. Right: by using the system, domain experts can accurately track features related to a synapse and details related to glycogen derived energy fluxes.

- the possibility of testing on the fly absorption hypothesis in order to plan and filter subsequent time-consuming statistical analysis;

- the combination of volume view and abstract layout view, which provides a way to inspect datasets in relatively short time and discover new absorption patterns, and visual correspondences between absorption patterns and morphology features.

Domain scientists particularly appreciated the way data is provided, since they could rapidly check and compare local and global features and they were able to inspect absorption patterns at various scales. From this point of view, the example shown in figure 11 right is emblematic, since it represents how the details of a synaptic contact are represented by our system. Neuroscientists the could easily trace all main structures, and they were also able to recognize specific features coming directly from Electron Microscopy data, like vesicles, mitochondria and endoplasmatic reticulum (ER). Neuroscientists also showed particular interest in tracking the shape of the astrocyte processes, and to relate the contact surface between them and neural processes and the highlights provided by the absorption maps, thus providing indication that astrocytes feed both synaptic end points through glycogen derived energy. Even if we designed the system for supporting ultrastructural analysis of neuroscience EM data, the positive feedback let us believe that similar strategies can be considered for the visualization of volumetric data coming from other application domains. In this case, the general requirements would be: a scalar volumetric field which can be subdivided in different labeled portions, and a static or dynamic intensity volumetric signal, that domain scientists want to interactively inspect concurrently with the original scalar field in a way to focus on regions where the intensity is higher.

\subsection{Comparison with other frameworks}

We asked domain experts to explore a subset of datasets in table 1 as to perform a qualitative comparison of the different features. Neuroscientists normally use standard surface visualization frameworks, like Blender, for performing visual inspection of neural structures decorated with glygogen distribution, in a way to speculate about proximity patterns and formulate hypothesis, even with the help of VR setups [CBB*16]. Recently, these surface rendering

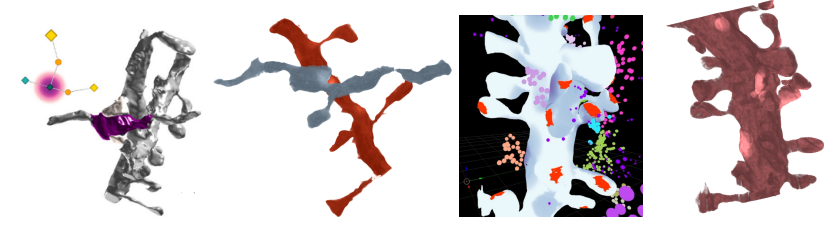

Figure 12: Comparison with Abstractocyte (top) [MAAB ${ }^{*}$ 18] and GLAM (bottom) [ABG* 18]: our framework can be considered complementary to both systems, but it provides more accurate absorption analysis, since the visualization uses directly EM data. Same feature visualized with both frameworks: at the top a Multi Synaptic bouton from Mouse 3 visualized with Abstractocyte (left), and with our system (right), at the bottom a dendrite from Hippocampus visualized with GLAM (left), and with our system (right).

frameworks were enriched by highlighting absorption on surface reconstructions (GLAM, proposed by Agus et al. [ABG* 18$]$ ), or by mapping glycogen content in abstract neurite representations ( $A b$ stractocyte, proposed by Mohammed et al. [MAAB $\left.\left.{ }^{*} 18\right]\right)$. After analysis sessions, neuroscientists concluded that all the frameworks can be considered complementary tools, since they provide different view of the same data. The main difference of our framework is that it works directly on EM volume data, that we use to compute the absorption information more accurately than the models proposed by Agus et al. $\left[\mathrm{ABG}^{*} 18\right]$ and Mohammed et al. [MAAB*18]. Figure 12 left shows similar structures represented with Abstractocyte [MAAB $\left.{ }^{*} 18\right]($ left) and the proposed framework (right): in this case a Multi Synaptic Bouton from mouse 3 connected to a dendrite. Domain scientists recognized that while the framework proposed by Mohammed et al. [MAAB*18] can be considered useful for general data overview, the proposed system is more accurate for detailed visual analysis. Specifically, our framework maintains the correct metric information and the morphology structure, in a way that local absorption patterns are accurately represented and insights can be extracted even about energy fluxes in small features of neural structures. Moreover, figure 12 right shows similar structures represented with GLAM [ABG*18](left) and the proposed framework(right): a dendrite from Hippocampus dataset with energy absorption mapped on the surface and on the volume. Domain scientists recognized that the main differences between these two systems are related to the way details are represented. Specifically, contrarily to the method proposed by Agus et al. [ABG*18], the proposed framework gives the scientist the chance to appreciate either the absorption peaks and the volumetric details of the structure, as they appear in the original EM data (like mitochondria and other organelles appearing as semitransparent dark artifacts during exploration).

\subsection{Use case: glycogen analysis for aging studies}

There is no need here to highlight how important is considered in neuroscience the study of aging mechanisms in brain. Since now, different structural studies have investigated how neuronal connectivity may be altered in the aging brain, but these have typically focused on particular morphology features like synapses [PSL08], dendrites [DWLH13], or axons [MAC*13, CWB*18a]. However, 

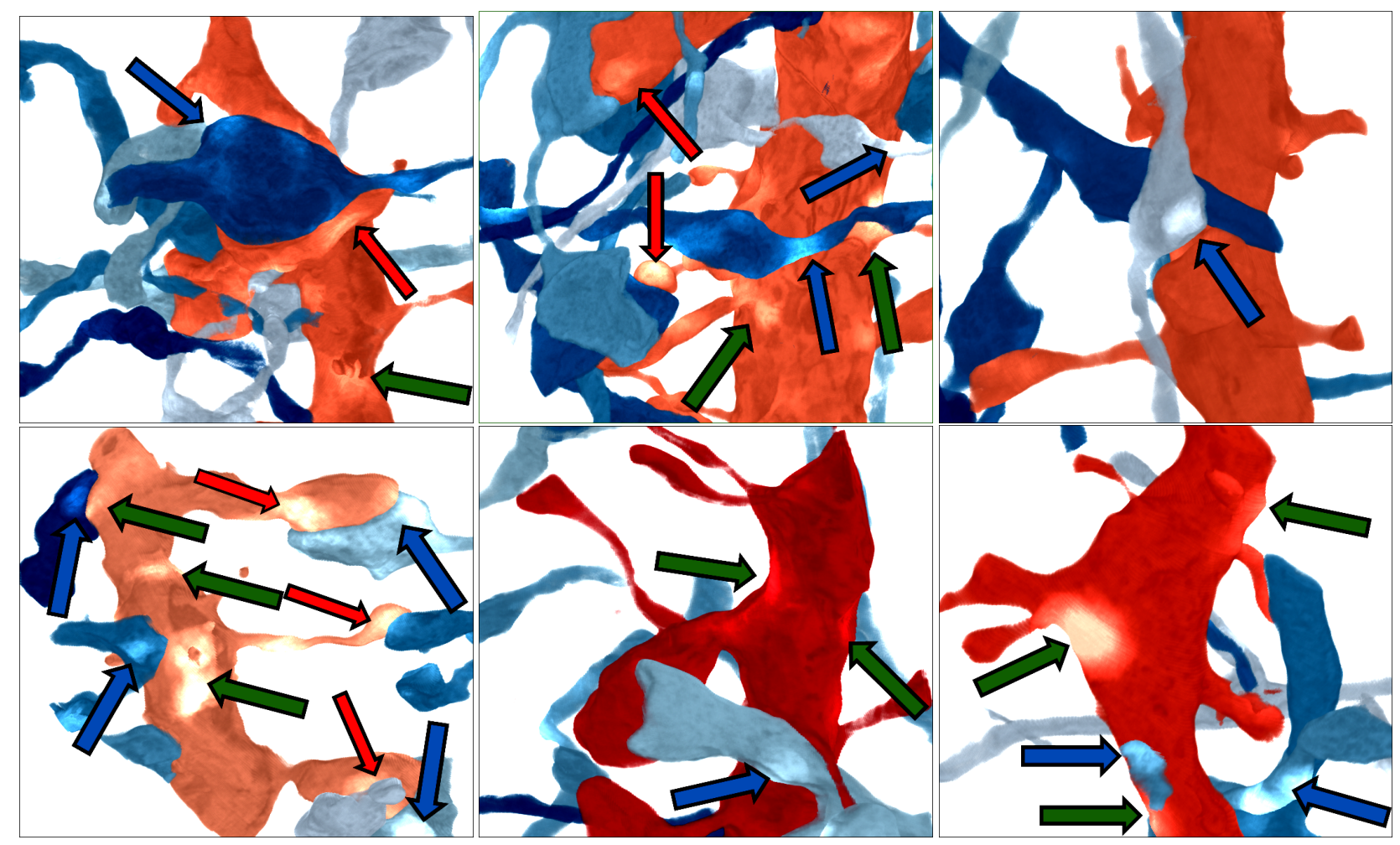

Figure 13: Snapshots from data inspection: features visualized with our frameworks on datasets in table 1. In order from top left to bottom right, from mouse1 to mouse6, one frame per data set is shown. Arrows indicate specific absorption patterns recognized by domain scientists: absorption peaks in boutons (in blue), in dendrite shafts (in green), and in synapses and spines (in red).

the effects of aging on glycogen content have not been yet explored adequately. Domain experts used the proposed system as preliminary tool for planning ad hoc statistical analysis of the six rodent somatosensory cortex datasets (three adults and three older) in table 1 [CWB* 18a]. After analysis, they visually inferred the following hypothesis (see figure 13):

- given a slight difference in synaptic density between mature ad elder animals [CWB*18a], as well as in spine density and size, through our system they could confirm that glycogen-derived energy is particularly needed to sustain synaptic activity and spine development and maintenance;

- through visual assessment of the graph layouts, and the inspection of absorption patterns at macroscopic scale, they got clues that glycogen-derived energy is absorbed by dendrites more than axons, as expected since dendrites contain more mitochocondria and ER, that contain the machinery for converting glycogenderived lactate to ATP, but contrarily to what observed in an adult rat hippocampus through glycogen analysis [CBB*16];

- they noticed various absorption peaks in dendrite shafts, in correspondence of mitochondria, indicating areas for creation of new spines (see green arrows in figure 13), that they supposed to be involved in memory formation process;

- they noticed a consistent number of absorption peaks around large synapses, especially in aging mice and in correspondence of bigger spines (see red arrows in figure 13), driving to the hy- pothesis that glycogen-derived energy is needed to sustain stable, more mature synaptic contacts;

- they noticed a significant number of absorption peaks around boutons, independently of the group, and especially multisynaptic ones (MSB) (see blue arrows in figure 13), indicating the possibility that those pre-synaptic terminals are in need of glycogen-derived energy. In particular, they noticed different absorption peaks in correspondence of different connection points.

As general outcomes, neuroscientists realized that there are perceivable differences in energy consumption between adult and elder animals: specifically, they have strong suspicion that while in adults most of energy is used for the spine and synapses development, in older animals most of the energy is used to sustain mature spines, bigger synapses, and boutons, especially multi-synaptic ones. We are currently investigating these speculations thoroughly under more rigorous statistical analysis.

\section{Conclusions}

We presented a framework for real time visual exploration of nanoscale electron microscopy volumes targeted to visual analysis of glycogen-derived energy absorption. The system integrates a high performance client-server architecture for volume visualization and a series of custom components for supporting specific metabolic analysis, including radiance-based absorption maps and node-link layout representation of neural connections, that enable 
accurate inspection at various scales of global and local absorption patterns during ultrastructural analysis. We successfully tested the system through qualitative evaluation by domain experts, which used the framework for planning statistical analysis of glycogen effects during aging in rodent brain samples. We plan to use the framework for supporting other studies involving glycogen effects in the central nervous system, like learning [ACD*18], the sleepwake cycle $\left[\mathrm{BdVK}^{*} 18\right]$, and the development of neurodegenerative diseases. Current limitations are related to data preprocessing which, at current stage, requires time-consuming human efforts, especially for dense reconstruction of neural structures from EM data $\left[\mathrm{CCK}^{*} 18\right]$. This partially limits the usage of the framework to ad hoc custom ultrastructural studies. We expect that in next future the processing pipeline will take advantage of machine learning techniques in a way that segmentation, dense reconstruction and metadata computation can be fully automatized [JKL*18]. To this end, we plan to invest resources in improving the reconstruction pipeline by considering automatic deep learning methods, with the expectation that artificial neural networks should perform best at mapping biological neural networks [FTG* 18]. In this way, most of the pipeline could be directly integrated into the digital acquisition process and appear completely transparent to domain scientists. Moreover, as pointed out by domain scientists, neural morphology visual comparisons are still considered complex and timeconsuming tasks. To this end, we plan to extend the framework by developing novel visual abstractions for supporting side-by-side comparisons of brain structures that involve specific simplified geometry representations, to be associated to $3 \mathrm{D}$ reconstructions. Finally, given the positive feedback from domain scientists, we plan to apply a similar strategy for inspection of static and dynamic intensity signals associated to volumetric data in other application domains, like simulation results in material science or geology.

Acknowledgments. This work was supported by the CRG Grant No. 2313 from King Abdullah University of Science and Technology KAUSTEPFL Alliance for Integrative Modeling of Brain Energy Metabolism. This work was conducted using resources and services at the Visualization Core Lab at KAUST. We also acknowledge the contribution of Sardinian Regional Authorities (project VIGECLAB). We thank Kalpana Kare and Danyia Boges, for the technical support; Graham Knott and the BioEM Facility at EPFL (Lausanne, Switzerland), for providing the EM stacks.

\section{References}

[AABH*16] Ai-AwAmi A. K., Beyer J., HaEhn D., Kasthuri N., Lichtman J. W., Pfister H., Hadwiger M.: Neuroblocks: Visual tracking of segmentation and proofreading for large connectomics projects. IEEE Trans. on Visualization and Computer Graphics 22, 1 (2016), 738-746. doi:10.1109/TVCG.2015.2467441.3

[AABS*14] Al-Awami A. K., Beyer J., Strobelt H., Kasthuri N., Lichtman J. W., Pfister H., Hadwiger M.: Neurolines: A subway map metaphor for visualizing nanoscale neuronal connectivity. IEEE Trans. on Visualization and Computer Graphics 20, 12 (2014), 2369-2378. doi:10.1109/TVCG.2014.2346312. 3

[ABG*18] Agus M., Boges D., Gagnon N., J.Magistretti P., HAdWIGER M., CALI C.: GLAM: Glycogen-derived Lactate Absorption Map for visual analysis of dense and sparse surface reconstructions of rodent brain structures on desktop systems and virtual environments. Computers \& Graphics 74 (August 2018), 85-98. doi: $10.1016 / \mathrm{j}$.cag.2018.04.007.2,3,4,5,8,10

[ACD*18] Alberini C. M., Cruz E., Descalzi G., Bessières B., GAO V.: Astrocyte glycogen and lactate: New insights into learning and memory mechanisms. Glia 66, 6 (2018), 1244-1262. doi:10.1002/ glia.23250. 12
[BAAK*13] Beyer J., Al-AwAmi A., KASThuri N., LichtMAN J. W., Pfister H., Hadwiger M.: ConnectomeExplorer: Queryguided visual analysis of large volumetric neuroscience data. IEEE Trans. on Visualization and Computer Graphics 19, 12 (2013), 28682877. doi:10.1109/TVCG.2013.142.3, 7

[BdVK*18] Bellesi M., De Vivo L., Koebe S., Tononi G., Cirelli C.: Sleep and wake affect glycogen content and turnover at perisynaptic astrocytic processes. Frontiers in Cellular Neuroscience 12, 308 (2018). doi:10.3389/fncel.2018.00308. 12

[BHP15] Beyer J., Hadwiger M., Pfister H.: State-of-the-art in GPU-based large-scale volume visualization. Computer Graphics Forum, 2015 34, 8 (Dec. 2015), 13-37. doi:10.1111/cgf.12605. 2

[BJA*19] Boorboor S., Jadhav S., Ananth M., Talmage D., RoLE L., KAUfMAN A.: Visualization of neuronal structures in widefield microscopy brain images. IEEE Trans. on Visualization and Computer Graphics 25, 1 (2019), 1018-1028. doi:10.1109/TVCG. 2018.2864852 .3

[BMA*19] Beyer J., Mohammed H., Agus M., Al-Awami A. K., Pfister H., HADWIGER M.: Culling for extreme-scale segmentation volumes: A hybrid deterministic and probabilistic approach. IEEE Trans. on Visualization and Computer Graphics 25, 1 (2019), 11321141. doi:10.1109/TVCG.2018.2864847.3,7

[BPH14] BRIX T., PRASSNI J.-S., HINRICHS K.: Visualization of large volumetric multi-channel microscopy data streams on standard PCs. arXiv preprint arXiv:1407.2074 (2014). 2

[BRGIG*14] Balsa Rodríguez M., Gobbetti E., Iglesias Guitián J., Makhinya M., Marton F., Pajarola R., Suter S. K.: State-of-the-art in compressed GPU-based direct volume rendering. Computer Graphics Forum 33, 6 (2014), 77-100. doi:10.1111/ cgf. 12280.2

[BSG*09] Bruckner S., Solteszova V., Groller E., Hladuvka J., BUhler K., JAI Y. Y., DiCKSON B. J.: Braingazer-Visual queries for neurobiology research. IEEE Trans. on Visualization and Computer Graphics 15, 6 (2009), 1497-1504. doi:10.1109/TVCG.2009. 121. 3

[Cal17] CALÌ C.: Astroglial anatomy in the times of connectomics. Journal of Translational Neuroscience 2, 4 (2017), 31-40. doi : 10 . 3868 / j.issn.2096-0689.2017.04.004.2

[CBB*16] Calì C., Baghabra J., Boges D. J., Holst G. R., Kreshuk A., Hamprecht F. A., SRINivasan M., LehVÄSlaiho H., Magistretti P. J.: Three-dimensional immersive virtual reality for studying cellular compartments in 3D models from EM preparations of neural tissues. Journal of Comparative Neurology 524, 1 (2016), 2338. doi:10.1002/cne.23852. 2, 8, 10, 11

[CCK*18] Coggan J. S., Calì C., Keller D., Agus M., Boges D., Abdellah M., Kare K., Lehväslaiho H., Eilemann S., JoLIVET R. B., ET AL.: A process for digitizing and simulating biologically realistic oligocellular networks demonstrated for the neuroGlio-Vascular ensemble. Frontiers in Neuroscience 12 (2018). doi: 10.3389 /fnins.2018.00664.5,12

[CSS*12] Cardona A., SaAlfeld S., Schindelin J., ArgandaCarreras I., Preibisch S., Longair M., TOMAnCAK P., HaRtenstein V., Douglas R. J.: Trakem2 software for neural circuit reconstruction. PloS one 7, 6 (2012), e38011. doi:10.1371/ journal.pone.0038011. 5

[CTM19] Calí C., TAuffenberger A., Magistretti P.: The strategic location of glycogen and lactate: From body energy reserve to brain plasticity. Frontiers in Cellular Neuroscience 13 (2019), 82. doi: 10.3389 /fncel.2019.00082. 2

[CWB*18a] Calì C., WaWrzyniak M., Becker C., Maco B., Cantoni M., Jorstad A., Nigro B., Grillo F., De Paola V., FUA P., ET AL.: The effects of aging on neuropil structure in mouse somatosensory cortex. a 3D electron microscopy analysis of layer 1 . PloS One 13, 7 (2018), e0198131. doi : 10.1371 / journal pone. $0198131.8,10,11$ 
[CWB*18b] Cali C., WaWrzYNiaK M., Becker C., Maco B. Cantoni M., Jorstad A., Nigro B., Grillo F., V D. P., P. F., G.W. K.: Data from: The effects of aging on neuropil structure in mouse somatosensory cortex. a 3D electron microscopy analysis of layer 1, 2018. doi:10.5061/dryad.bh78sn5. 2, 8, 9

[DH96] Davidson R., HAREL D.: Drawing graphs nicely using simulated annealing. ACM Trans. on Graphics 15, 4 (1996), 301-331. doi:10.1145/234535.234538.6,7

[DRN*17] Díaz J., Ropinski T., NAVAzo I., GobBetTi E., VÁZQUEZ P.-P.: An experimental study on the effects of shading in 3D perception of volumetric models. The Visual Computer 33, 1 (2017), 47-61. doi:10.1007/s00371-015-1151-6.3

[DWLH13] Dickstein D. L., Weaver C. M., LuebKe J. I., Hof P. R.: Dendritic spine changes associated with normal aging. Neuroscience 251 (2013), 21-32. doi:10.1016/j.neuroscience. 2012.09 .077 .10

[FTG*18] Funke J., Tschopp F. D., Grisaitis W., Sheridan A., Singh C., SAAlfEld S., Turaga S. C.: Large scale image segmentation with structured loss based deep learning for connectome reconstruction. IEEE Trans. on Pattern Analysis and Machine Intelligence (2018). doi:10.1109/TPAMI.2018.2835450. 12

[HAAB*18] Hadwiger M., Al-Awami A. K., Beyer J., Agus M., PFISTER H.: Sparseleap: Efficient empty space skipping for large-scale volume rendering. IEEE Trans. on Visualization and Computer Graphics 24, 1 (2018),974-983. doi:10.1109/TVCG.2017.2744238.3, 7

[HB03] Harrower M., Brewer C. A.: Colorbrewer. org: an online tool for selecting colour schemes for maps. The Cartographic Journal 40, 1 (2003),27-37. doi:10.1179/000870403235002042.8

[HBJP12] Hadwiger M., Beyer J., JeOng W.-K., Pfister H.: Interactive volume exploration of petascale microscopy data streams using a visualization-driven virtual memory approach. IEEE Trans. on Visualization and Computer Graphics 18, 2 (2012), 2285-2294. doi : $10.1109 /$ TVCG.2012.240.2,3,7

[HCT*17] Hildebrand D. G. C., Cicconet M., Torres R. M. Choi W., Quan T. M., Moon J., Wetzel A. W., Champion A. S., GRAHAM B. J., RANDLETT O., ET AL.: Whole-brain serial-section electron microscopy in larval zebrafish. Nature 545, 7654 (2017), 345. doi: 10.1038/nature22356.2

[HWP*18] Haleem H., Wang Y., Puri A., Wadhwa S., QU H.: Evaluating the readability of force directed graph layouts: A deep learning approach. arXiv preprint arXiv:1808.00703 (2018). 6

[JKL*18] JANUSZEWSKI M., KORNFELD J., Li P. H., POPE A., Blakely T., Lindsey L., Maitin-Shepard J. B., TyKa M., DenK W., JAIN V.: High-precision automated reconstruction of neurons with flood-filling networks. Nature methods 15, 8 (2018), 605. doi: $10.1038 / \mathrm{s} 41592-018-0049-4.12$

[JSYR12] JÖNSSON D., SUNDÉn E., YNNERMAN A., ROPINSKI T.: State of the art report on interactive volume rendering with volumetric illumination. In Eurographics 2012 (2012), Eurographics-European Association for Computer Graphics, pp. 53-74. doi:10.2312/conf/ EG2012/stars/053-074.3

[LPS14] Lichtman J. W., Pfister H., Shavit N.: The big data challenges of connectomics. Nature Neuroscience 17, 11 (2014), 1448 doi: $10.1038 / \mathrm{nn} .3837 .2,3$

[LSPV15] Lawonn K., Smit N. N., Preim B., Vilanova A.: Illustrative multi-volume rendering for PET/CT scans. In Proc. VCBM (2015), pp. 103-112. doi :10.2312/vcbm. 20151213. 3

[MA15] Magistretti P. J., Allaman I.: A cellular perspective on brain energy metabolism and functional imaging. Neuron 86, 4 (2015), 883-901. doi:10.1016/j.neuron.2015.03.035. 2, 3, 5

[MAAB*18] Mohammed H., Al-Awami A. K., Beyer J., Cali C., Magistretti P., Pfister H., Hadwiger M.: Abstractocyte: A visual tool for exploring nanoscale astroglial cells. IEEE Trans. on Visualization and Computer Graphics 24, 1 (2018), 853-861. doi: $10.1109 /$ TVCG. 2017.2744278. 2, 3, 4, 10
[MAC*13] Mostany R., Anstey J. E., Crump K. L., Maco B., KNotT G., Portera-CAilliaU C.: Altered synaptic dynamics during normal brain aging. Journal of Neuroscience 33, 9 (2013), 4094-4104. doi:0.1523/JNEUROSCI . 4825-12.2013. 10

[MGLS*16] MERTEN N., Glasser S. LASSEN-SCHMIDT B., Grosser O. S., Ricke J., Amthauer H., Preim B.: Illustrative PET/CT visualisation of SIRT-treated lung metastases. In Proc. VCBM (2016), pp. 99-103. doi:10.2312/vcbm.20161276. 3

[NOE*10] NGuYen T. K., Ohlsson H., EKLund A., Hernell F., Ljung P., Forsell C., Andersson M., Knutsson H., YNNERMAN A.: Concurrent volume visualization of real-time fMRI In 8th IEEE/EG International Symposium on Volume Graphics, Norrköping, Sweden, 2-3 May, 2010 (2010), Eurographics-European Association for Computer Graphics, pp. 53-60. doi:10.2312/VG/VG10/ $053-060.3$

[OBA*16] OE Y., Baba O., Ashida H., NaKamura K. C., Hirase H.: Glycogen distribution in the microwave-fixed mouse brain reveals heterogeneous astrocytic patterns. Glia 64, 9 (2016), 1532-1545. doi : 10.1002 /glia.23020.2,9

[OZ17] OUYANG W., ZIMmer C.: The imaging tsunami: Computational opportunities and challenges. Current Opinion in Systems Biology 4 (2017), 105-113. doi:10.1016/j.coisb.2017.07.011. 2

[PBC*16] Preim B., BAER A., CUNningham D., IsenberG T., ROPINSKI T.: A survey of perceptually motivated 3D visualization of medical image data. Computer Graphics Forum 35, 3 (2016), 501-525. doi:10.1111/cgf.12927.3

[PSL08] PeTers A., Sethares C., Luebke J : Synapses are lost during aging in the primate prefrontal cortex. Neuroscience 152, 4 (2008), 970-981. doi:10.1016/j.neuroscience.2007.07.014.10

[SBS*13] Sorger J., Bühler K., Schulze F., LiU T., Dickson B.: NeuroMAP-Interactive graph-visualization of the fruit fly's neural circuit. In 2013 IEEE Symposium on Biological Data Visualization (BioVis) (2013), IEEE, pp. 73-80. doi:10.1109/BioVis. 2013. 6664349.3

[SCRL16] Sarton J., Courilleau N., Remion Y., LuCAS L.: Towards an interactive navigation in large virtual microscopy images on 3D displays. In 3D Imaging (IC3D), 2016 International Conference on (2016), IEEE, pp. 1-5. doi:10.1109/IC3D.2016. 7823463.2

[SMB*17] Swoboda N., Moosburner J., Bruckner S., Yu J., DICKSON B. J., BÜHLER K.: Visualization and quantification for interactive analysis of neural connectivity in drosophila. Computer Graphics Forum 36, 1 (2017), 160-171. doi:10.1111/cgf.12792. 3

[SSKH11] Sommer C., Straehle C., Köthe U., Hamprecht F. A.: Ilastik: Interactive learning and segmentation toolkit. In 2011 IEEE international symposium on biomedical imaging: From nano to macro (2011), IEEE, pp. 230-233. doi:10.1109/ISBI.2011. 5872394.5

[TG16] TitzE B., Genoud C.: Volume scanning electron microscopy for imaging biological ultrastructure. Biology of the Cell 108, 11 (2016), 307-323. doi:10.1111/boc.201600024.8

[ZL13] ZHU C., LIU Q.: Review of monte Carlo modeling of light transport in tissues. Journal of Biomedical Optics 18, 5 (2013), 050902 050902. doi:10.1117/1.JBO.18.5.050902.5

[ZLP* 18] Zheng Z., LAURITZEN J. S., Perlman E., RobinSON C. G., Nichols M., Milkie D., Torrens O., Price J., Fisher C. B., Sharifi N., ET AL.: A complete EM volume of the brain of adult drosophila melanogaster. Cell 174, 3 (2018), 730-743. doi: $10.1016 / j . c e l 1.2018 .06 .019 .2$

[ZPG17] Zheng J. X., PAWAR S., Goodman D. F.: Graph drawing by stochastic gradient descent. arXiv preprint arXiv:1710.04626 (2017). 6, 